\title{
Framework of simulation experiment factors screening for equipment system
}

\author{
YANG Ning-meng ${ }^{1, a, *}$, YANG Fan-de $e^{1, b}$ \\ ${ }^{1}$ Academy of Equipment, Huairou, Beijing, China \\ alemony2016@163.com, byangfd5908@sina.com \\ *Corresponding author
}

Keywords: Complex equipment system, Simulation experiment, Experiment index, Factor screening.

\begin{abstract}
The characteristic of the equipment system's complexity makes the simulation facing the problem of massive factors, easily lead to combination blasting on the simulation experiment design, and then put forward the framework of screening factor for equipment simulation experiment system. First of all, the characteristics of the simulation experiment of the equipment system are analyzed, and the simulation index is determined from the user's point of view. Secondly, the factor sample space is studied, and the relevant factors are selected according to the experiment index, factors are normalized next. Then select the appropriate screening method to extract the important factors for the sample space and specific problems, and finally verify. Based on the analysis of the experiment index, the study of the sample space, the extraction and validation of the important factors, a set of complete screening scheme is proposed, it has certain significance for the design of equipment system simulation experiment.
\end{abstract}

\section{Introduction}

With the development of information technology and the future war, the equipment system becomes more and more complicated and diversified, and it also has become more closely. The evaluation of the equipment system is an effective way to judge the effectiveness of the equipment system. At present, there are many methods to evaluate the equipment system, and the simulation experiment is one of the most common methods. In order to ensure the credibility of the simulation results, the simulation experiment of the equipment system should take into account its own performance, environmental impact, and so on. However, due to the complexity of the equipment system, it is very difficult to consider all the factors, it is a waste of time and waste of manpower and material resources. The main concern is the simulation modeling at present, and there are few researches on the simulation design, especially screening the factors in the design stage. According to Pareto or 20-80 rule ${ }^{[1]}$, only a small number of important factors have a significant impact on the experiment index. Therefore, it is very important to select the important factors for the simulation experiment. The reasonable selection of factors is related to the reliability of simulation results. At present, in the logistics system ${ }^{[2]}$, supply chain ${ }^{[3]}$ and other application fields have been carried out factor screening research. However, the research on the selection of factors for the operational 
effectiveness evaluation of complex equipment system or equipment system is still scarce. In this paper, based on the background of the efficiency evaluation of complex equipment system, the selection of simulation experiment factors is studied, as shown in Figure 1, it has certain significance to optimize the simulation experiment design.

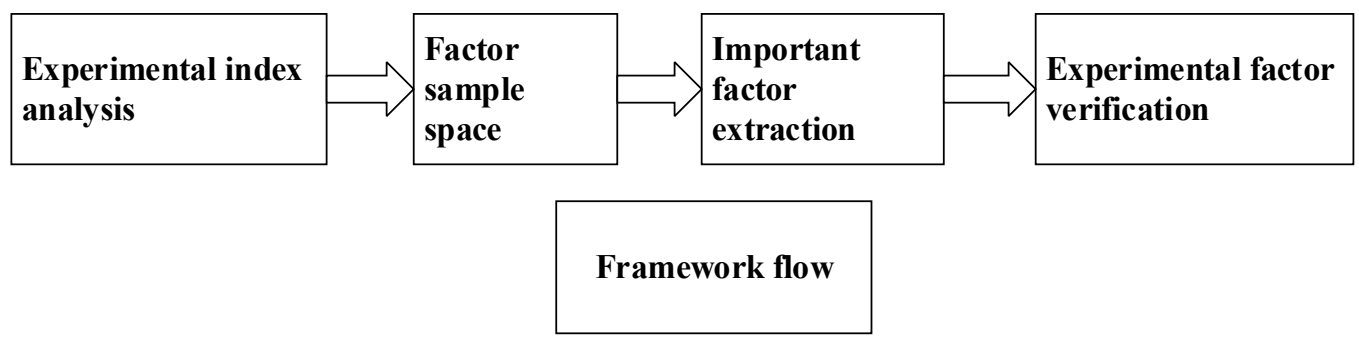

Figure 1 Framework flow

\section{Experiment index analysis}

The experiment index is the object of the whole screening process, and it is the basis for the selection of factors. It is the key prerequisite for the development of factor screening at the same time, and it also must be identified before screening for important factors.

The factors are directly related to the experiment index. Generally speaking, it is based on the determination of the experiment index, and then causal backtracking, select the factors may have an impact on the experiment index. In the simulation experiment of the equipment system, the experiment index is considered by the designer in the user's point of view of the equipment system, which is the ability that the user wants to realize or achieve. There are several principles to select the experiment index:

(1) Objectivity. The selection of experiment index must be objective, which is practical and needs to be considered;

(2) Rationality. According to the purpose of the experiment, the selection of the experiment index needs to be reasonable, and it should be considered for the purpose of the experiment, and the index which has nothing to do with the experiment can not be chosen;

(3) Sensitivity. The selection of the experiment index is easier to cause fluctuations according to the change of input, the change value is easy to observe;

(4) Interchangeability. When the selected experiment indicators can not be seen directly from the experimental results, it can be seen through mathematical transformation and other methods.

\section{Factor sample space}

The factor is defined as the controllable input variable, its change can affect the change of the output. The factor is the object in the process of screening, the selected important factors will be used in the design of simulation experiment directly. Experiment factors are the foundation and key elements of equipment system simulation design.

\subsection{Main effect and interaction}

(1) Main effect

The main effect of the factor is to change the input of the factor to make the output has a maximum value. If the influence of the factor on the output is monotonic, then the absolute value of the difference between the high and low level and the corresponding output value is the main effect of the factor on the output. 


\section{(2) Interaction}

The interaction between factors: the influence of one factor on the experiment index will be different according to the change of the other factor level, which is called the interaction between the two factors. Such as factor A interacts with B, denoted as A*B or AB.

(3) Main effect direction

The direction of the main effect is used to measure the positive and negative effects of the factors on the output. With the increase of the input level of experimental factors, the output value increased by positive expressed. Obviously, if the influence of the factor on the output is monotonically increasing, the direction of the main effect is positive; if the influence of the factor on the output is monotonic, then the direction of the main effect is negative.

\subsection{Classification of factors}

In the simulation experiment of equipment system, the classification of factors can be considered from two aspects:

(1) From the factor level, they can be divided into discrete type, continuous type, extreme type, that is, the different state of the factor, the discrete variable or the continuous variable, or on the boundary of the factor state;

(2) From the composition of factors, they can be divided into: environment; strategy; interoperability; performance. Environmental factors that are related to the environment when using equipment system, such as sunny, rain, snow and so on. Strategy is the strategy used when using the equipment system, such as the composition of the structure of the equipment system, the use of order, etc. interoperability is the subsystem interconnection capability when using the equipment system, such as data reading, storage. Performance is the characteristic of the equipment system itself, such as the ability of the radar detection range, fighter combat radius.

After the determination of the experiment index, the top-down decomposition method will used to find the possible factors that might influence the experiment index. The selection of the factors has the following requirements ${ }^{[4]}$ :

(1) Objective and comprehensive. The factors for the selection of experiment indicators must be objective, not subjective imagination. The choice of the factors can be qualitative analysis. Select the factors should be taken into account comprehensively, and do not miss any factor.

(2) Key, independent and sensitive. The factors should have a key influence on the experimental index, and the factors should be as independent as possible to reduce the interaction between the factors.

\subsection{The construction of the sample space}

Since this paper is oriented to the object of the equipment system, without considering the human factors, only from the environmental factors, strategic factors, performance factors and interoperability considerations.

(1) Environmental factors: the collection of all external factors that affect the simulation system;

(2) Strategic factors: the collection of equipment system use strategies;

(3) Performance factors: the collection of key performance indicators in the system;

(4) Interoperability: refers to the impact of the various subsystems of the equipment system interconnection index collection.

The factors that have been chosen should be uniformed, each factor needs to be sorted and numbered. In accordance with the number, the name of the factors, the basic level, high level, low level list, for example Table 1. 
Table 1 Uniform sample

\begin{tabular}{|c|c|c|c|c|c|}
\hline No. & Variables & Unit & Base value & High level (+) & Low level (-) \\
\hline 1 & $\begin{array}{c}\text { Launch } \\
\text { quantity }\end{array}$ & PCS & 10 & 16 & 8 \\
\hline 2 & CEP & $\mathrm{m}$ & 200 & 300 & 100 \\
\hline 3 & Power radius & $\mathrm{m}$ & 200 & 300 & 100 \\
\hline$\ldots$ & $\ldots$ & $\ldots$ & $\ldots$ & $\ldots$ & $\ldots$ \\
\hline
\end{tabular}

\section{Important factor extraction}

After the establishment of factor sample space, combined with the size of the factor and the experiment times, the appropriate factor screening method will be selected. The screening method and simulation model are combined to extract the important factors through the simulation data analysis.

Factor screening methods can be broadly divided into two categories: one is for a small number of factors, and the other is for a large number of factors. The method that is suitable for a small number of factors is factorial design, and the sequential bifurcation is more suitable for a large number of factors.

\subsection{Screening methods}

\subsubsection{Factorial design method}

In the 20s and 30s of last century, Fisher had put forward the concept of factorial design based on agricultural statistics, factorial design can be divided into full factorial design and partial factorial design. Due to the small number of factors, factorial design is a very effective method. $2^{k}$ factorial design method is a special kind of factorial design, in which the number of factors is $\mathrm{k}$, and each factor has only two levels, therefore, the time of experiments required is $2^{k}$. When the number of factors is relatively large, there is a large amount of computation in the $2^{k}$ factorial design. In order to improve the efficiency of experiment, $2^{k-p}$ fractional factorial design is proposed, where $\mathrm{k}$ is the total number of factors, factor k-p required for the experiment, so the number of experiments is $2^{k-p}$, the main steps are:

(1) Determine the number, level and resolution of the factors, each factor is set to two levels, that are, high and low levels;

(2) Select the k-p factors in accordance with the high level of +1 , the low level of -1 and design factor matrix;

(3) When the design of the matrix is completed, according to the calculation and analysis method in the full factorial design, the main effect and the interaction between the factors will be calculated.

$2^{k}$ factorial design and $2^{k-p}$ fractional factorial design are mainly aimed at the factors of a few advantages.There is no need to consider the factor's monotonicity and main effect. The main drawback is that the factor number once increased, it will lead to the experiment times increase exponentially.

\subsubsection{Sequential Bifurcation}

Due to the simulation object is more and more complex equipment system, the multi factors screening method is introduced, that is the group screening method. A series of methods, which are represented by sequential bifurcation ${ }^{[5]}$, are used more. The SB puts all factors into the same group 
firstly, then determine whether there is an important factor in this group. If there is, continue to divide the factors into two groups, and determine whether there is an important factor respectively; if not, the group will be dropped, and continue grouping. Until the unimportant factors are kicked out and the important factors are extracted. For example, the 16 factors were selected, as shown in Figure 2.

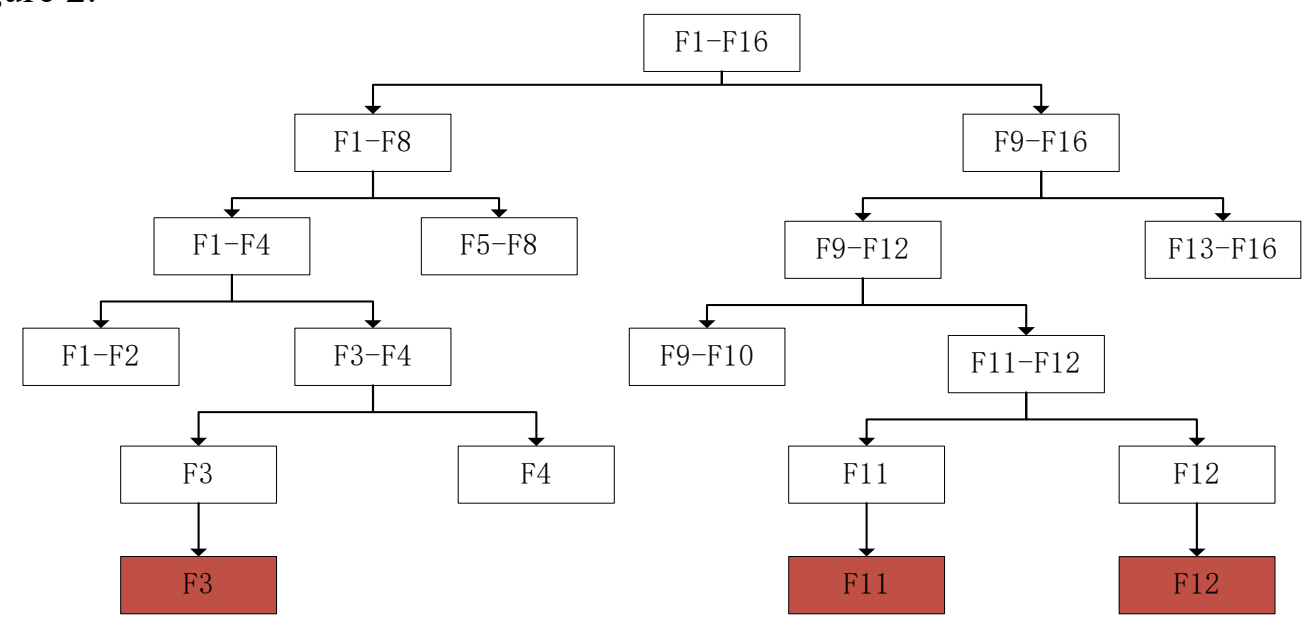

Figure 2 Screening sample

Based on the SB method, without considering the factor of interaction between cases, there are two assumptions ${ }^{[6]}$ :

(1) The input and output of the simulation experiment is represented by a first order element model:

$$
\mathrm{Y}=\beta_{0}+\beta_{1} x_{1}+\beta_{2} x_{2}+\ldots+\beta_{j} x_{j}+e
$$

$\mathrm{Y}$ is the output, $x_{j}$ is the value of the $\mathrm{j}$, the high level values of 1 low values of $-1, \beta_{j}$ represents the main effect of factor $j$, and e represents the error.

(2) As long as there is the main effect of each factor, the main effect sign of the factor is known.

Now define the following symbols and give meaning:

$Y(\mathrm{j}) ; \mathrm{r}$ : where $\mathrm{r}=1,2 \ldots$, is the simulated output, in which, $x$ from $x_{1}$ to $x_{j}$ take +1 , the remaining factors take $-1, \quad \beta$ from $\beta_{0}$ to $\beta_{j}$ take a high level, the remaining take the low level;

$\beta_{i-j}$ : the sum of main effect from factors $\mathrm{i}$ to $\mathrm{j}$;

$$
\beta_{i-j}=\sum_{k=i}^{j} \beta_{k}
$$

The sum of main effect from factors $i$ to $j$ in $r$ replicate:

$$
\hat{\beta}_{i-j ; r}=\frac{Y(\mathrm{j}) ; \mathrm{r}-\mathrm{Y}(\mathrm{i}-1) ; \mathrm{r}}{2}
$$

The main effect of the single factor in $r$ replicate is:

$$
\hat{\beta}_{j ; r}=\frac{Y(\mathrm{j}) ; \mathrm{r}-\mathrm{Y}(\mathrm{j}-1) ; \mathrm{r}}{2}
$$

Note that these equations are established only when the interaction between the factors is not considered, and the average population and the average single factor main effects are calculated as follows:

$$
\hat{\beta}_{i-j}=\sum_{r=1}^{m} \hat{\beta}_{i-j ; r}
$$




$$
\overline{\hat{\beta}_{j}}=\sum_{r=1}^{m} \hat{\beta}_{j ; r}
$$

In addition to SB, there are many improved methods of SB method and other methods won't be introduced, this paper lists several commonly used multi factor screening methods ${ }^{[7]}$, as shown in the table 2 .

Table 2 Comparison of screening methods

\begin{tabular}{|c|c|c|c|c|}
\hline methods & efficiency & \multicolumn{2}{|c|}{ robustness } & assumption \\
\hline Running time & $\begin{array}{c}\text { Monotone } \\
\text { property }\end{array}$ & $\begin{array}{c}\text { Number } \\
\text { of } \\
\text { Variables }\end{array}$ & $\begin{array}{c}\text { the main } \\
\text { effect } \\
\text { direction }\end{array}$ \\
\hline $\begin{array}{c}\text { Sequential } \\
\text { Bifurcation }\end{array}$ & $\mathrm{O}\left(K \log _{2} K\right)$ & Yes & Large & Yes \\
\hline Trocine Screening & Varies & No & Medium & No \\
\hline IFFD & $O(K)$ & No & Large & No \\
\hline $\begin{array}{c}\text { Two-Stage Group } \\
\text { screening }\end{array}$ & Varies & Yes & Medium & Yes \\
\hline
\end{tabular}

\subsection{Screening processes}

In order to provide guidance for the screening of the equipment system simulation experiment factors, the factor screening process model is given, as shown in Figure 3.

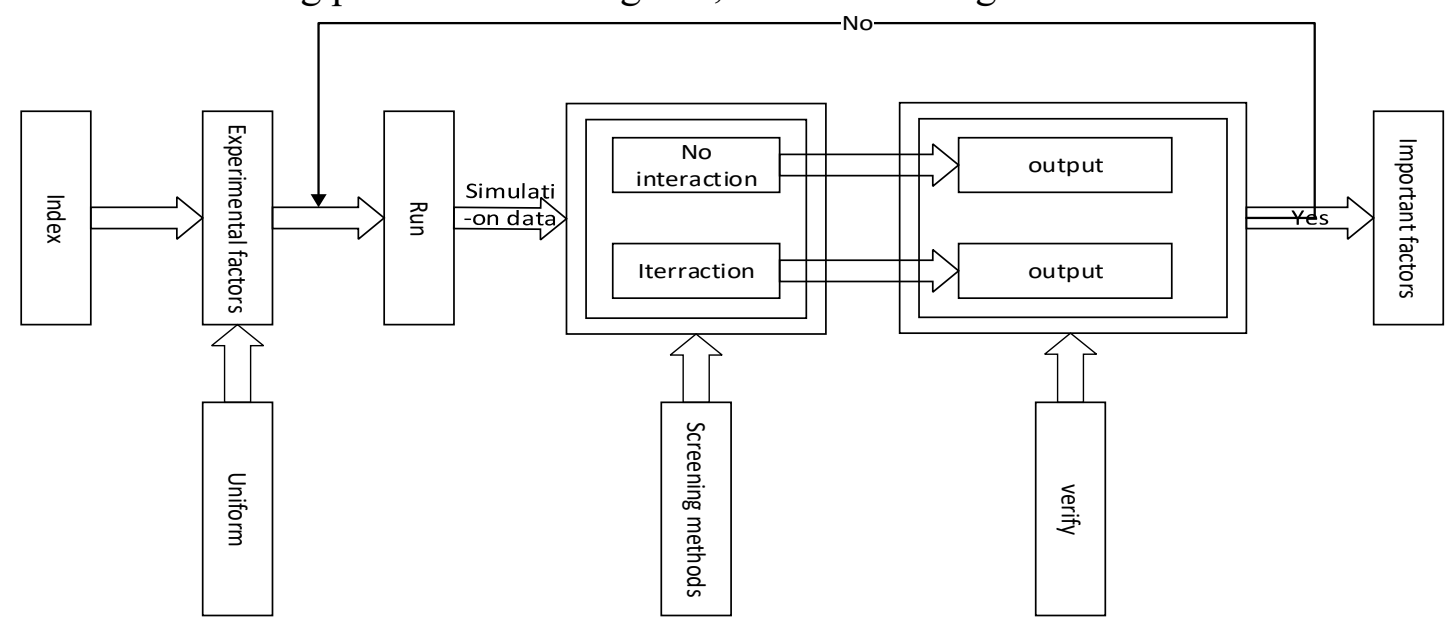

Figure 3 Screening process model

In view of the above process model, the details are as follows:

(1) From the point of view of the users of the equipment system, according to the content and the principle of selection, select the appropriate indicators;

(2) Top-down analysis, selection of factors that may have an impact on the parameters, and uniform specifications;

(3) According to the number of factors, the experiment times, whether or not to consider the interaction between the factors and the resolution of the situation, select the appropriate factor screening method; 
(4) Simulation experiment was carried out to obtain the corresponding output data;

(5) Using the factor screening method to extract the factors;

(6) Based on the experimental efficiency and two kinds of errors, the important factors are validated, if the requirement is satisfied, it is an important factor, or repeat (2) - (6) steps, until the important factors are found.

\section{Factor verification}

\subsection{Factor validation index}

The verification of factors can be considered in the following two aspects:

(1) Efficiency. Efficiency is measured by the number of experiments carried out by simulation experiments, the less the number of running experiments, the higher the efficiency.

(2) Effectiveness. The effectiveness of the evaluation criteria is to ensure that the elimination of unimportant factors, and important factors are retained. There are two kinds of errors in the selection of the important factors.

Class I error: When the factors are selected, the unimportant factors are extracted as important factors.

The expression is calculated : $E_{1}=\frac{N_{\text {Unimportant-Factors }}}{N_{\text {All Factors }}}$

Class II error: factor screening method does not filter the important factors, the important factors are missing.

The expression is calculated : $E_{2}=\frac{N_{\text {Missing Important-Factors }}}{N_{\text {All Factors }}}$

In general, the severity of type II errors is much greater than class I errors. Class I errors will only increase the complexity of the simulation experiments, the amount of calculation and other issues, will not cause a conclusion error. However, the emergence of class II error will lead to the wrong conclusion and the whole simulation experiment will be failed.

\subsection{Verification}

Unimportant factors can be verified through two processes after the important factors were extracted.Two specific processes $^{[8]}$ :

(1) Two times running in the simulation model, the first run, all factors are placed in the high level, the output is $W_{1}$; the second run ,all the factors in the low level, the output is $W_{2}$. The difference of the two run output $\delta_{1}=W_{1}-W_{2}$.

(2) The same for the two times running in the simulation model, the first run, the important factors placed in the high level, the important factors placed in the low level, then the output is $W_{3}$; in the second run, the important factors will place in the low level, and the unimportant factors will place in the high level, the output is $W_{4}$. As a result, $\delta_{2}=W_{1}-W_{3}, \delta_{3}=W_{2}-W_{4}$.

(1) If $\delta_{1}$ is large, both of $\delta_{2}$ and $\delta_{3}$ are small, the unimportant factors are really unimportant;

(2) If $\delta_{1}, \delta_{2}, \delta_{3}$ are small, all the factors are not important;

(3) If $\delta_{1}$ is large, one or both of $\delta_{2}$ and $\delta_{3}$ are large, there will be important factors in the unimportant factors;

(4) If $\delta_{1}$ is small, one or both of $\delta_{2}$ and $\delta_{3}$ is large, there may be some problems in the definition 
of important and unimportant factors.

\section{Conclusion}

When simulating the complex equipment system, specific simulation methods are needed to analyze and design the factors. In general, according to the simulation process of complex equipment system, the number of factors, the experiment times, the manpower and material resources and the time cost should be considered, and appropriate factor screening method will be selected. The further study on selecting simulation experiment factors for complex equipment system should be improved. The factor screening method suitable for complex equipment system needs to be further explored.

\section{References}

[1] Tuy H, Ricceri B, Frenk J B G, et al. Pareto optimality, game theory and equilibria[M]. Springer New York, 2008.

[2] Shi W, Shang J, Liu Z, et al. Optimal design of the auto parts supply chain for JIT operations: Sequential bifurcation factor screening and multi-response surface methodology[J]. European Journal of Operational Research, 2014, 236(2):664-676.

[3] Shi W, Liu Z, Shang J, et al. Multi-criteria robust design of a JIT-based cross-docking distribution center for an auto parts supply chain[J]. European Journal of Operational Research, 2013, 229(3):695-706.

[4] Zhu S, Li Z, Hu X, et al. Simulation Experiment Result Forecast Method Based on Support Vector Machine $[\mathrm{C}] / /$ International Conference on Intelligent Computation Technology and Automation. IEEE Xplore, 2009:220-223.

[5] Kleijnen J P C, Bettonvil B, Persson F. Screening for the Important Factors in Large Discrete-Event Simulation Models: Sequential Bifurcation and Its Applications[J]. Ssrn Electronic Journal, 2003, 2003-104(2003-104):287-307.

[6] Ragheb M, Rekab K, Tadj, et al. Screening alexandria port simulation model by using sequential bifurcation procedure[J]. International Journal of Simulation Modelling, 2010, 9(2):98-109.

[7] Trocine L, Malone L C. Finding important independent variables through screening designs:a comparison of methods[C]// wsc. IEEE Computer Society, 2000:749-754.

[8] Cioppa T M. An efficient screening methodology for a priori assessed non-influential factors[C]// Simulation Conference, 2004. Proceedings of the. IEEE, 2004:699-703. 